\title{
Correction to: Practical indications for the prevention and management of SARS-CoV- 2 in ambulatory dialysis patients: lessons from the first phase of the epidemics in Lombardy
}

\author{
Giuseppe Rombolà ${ }^{1}$ - Marco Heidempergher ${ }^{2}$. Luciano Pedrini ${ }^{3}$. Marco Farina ${ }^{4}$. Filippo Aucella ${ }^{5}$. \\ Piergiorgio Messa ${ }^{6}$. Giuliano Brunori ${ }^{7}$
}

Published online: 30 March 2020

(C) Italian Society of Nephrology 2020

\section{Correction to: Journal of Nephrology https://doi.org/10.1007/s40620-020-00727-y}

The originally published article the co-author name Marco Heidempergher incorrectly spelled as Marco Hedemperger. The correct name given below:

Marco Heidempergher.

The original article has been corrected.

The original article can be found online at https://doi.org/10.1007/ s40620-020-00727-y.

Giuseppe Rombolà

giuseppe.rombola@asst-settelaghi.it

1 Sezione Lombarda SIN, U.O. Nefrologia, Dialisi e Trapianto, ASST Sette Laghi, Varese, Italy

2 Sezione Lombarda SIN, U.O. Nefrologia e Dialisi, ASST FBF Sacco, Milan, Italy

3 U.O. Nefrologia e Dialisi, Ospedale Bolognini ASST Bergamo Est, Bergamo, Italy

4 U.O. Nefrologia e Dialisi, ASST Lodi, Lodi, Italy

5 Società Italiana di Nefrologia, U.O. Nefrologia e Dialisi, IRCCS “Casa Sollievo della Sofferenza” San Giovanni Rotondo, Foggia, Italy

6 Società Italiana di Nefrologia-SIN, U.O. Nefrologia, Dialisi e Trapianto, Ospedale Ca Granda Milano, Milan, Italy

7 Società Italiana di Nefrologia-SIN, U.O. Nefrologia e Dialisi, Ospedale Santa Chiara, Trento, Italy
Publisher's Note Springer Nature remains neutral with regard to jurisdictional claims in published maps and institutional affiliations. 